\title{
Digitale Radiografie in der Untersuchung von arbeits- und umweltbedingten Lungen- und Pleuraerkrankungen Anpassung der ILO-Kodierung
}

\author{
Digital Radiography in Chest Imaging of Occupational and Environmental Lung Diseases \\ Adaptation of the ILO Code
}

Autoren

Institute
K. G. Hering ${ }^{1}$, E. Borsch-Galetke², H. J. Elliehausen ${ }^{3}$, K. Frank ${ }^{4}$, H. G. Hieckel ${ }^{5}$, K. Hofmann-Preiß ${ }^{6}$, W. Jacques ${ }^{7}$, U. Jeremie ${ }^{8}$, N. Kotschy-Lang ${ }^{9}$, T. Kraus ${ }^{10}$, E. Mannes ${ }^{11}$, H. Otten ${ }^{12}$, W. Raab ${ }^{13}$, H. J. Raithel ${ }^{14}$, W. D. Schneider ${ }^{15}$, S. Tuengerthal ${ }^{16}$

Die Institutsangaben sind am Ende des Beitrags gelistet. eingereicht 11.8. 2009

akzeptiert nach Revision 18. 8. 2009

Bibliografie

DOI http://dx.doi.org/

10.1055/s-0029-1215110

Online-Publikation: 27. 10. 2009

Pneumologie 2009; 63:

664-668 @ Georg Thieme

Verlag KG Stuttgart · New York

ISSN 0934-8387

Korrespondenzadresse

Dr. med. Kurt Georg Hering

Knappschaftskrankenhaus

Dortmund

Wieckesweg 27

44309 Dortmund

K.G.Hering@t-online.de

\section{Zusammenfassung \\ $\nabla$}

Die Beurteilung der Pneumokoniose im Rahmen von Vorsorge und Begutachtung nach den Kriterien der ILO und unter Verwendung der von der ILO publizierten Vergleichsfilme hat in Deutschland eine lange Tradition. Die weite Verbreitung der digitalen Radiografie stellt die arbeitsmedizinische und pneumologische Praxis zunehmend vor Probleme, da weder die technischen Anforderungen hinreichend bekannt sind noch die erforderliche Ausstattung in der Praxis zur Verfügung stehen. Die vorliegende Arbeit gibt den aktuellen Stand zum Vorgehen bei der Erstellung, Befundung und Dokumentation digital gefertigter Röntgenaufnahmen im Rahmen der Untersuchung von Lunge und Pleura wieder.

\section{Einleitung}

$\nabla$

Der Übergang von der analogen zur digitalen Radiografie erfordert eine zeitnahe Anpassung der röntgenologischen Befundung und Dokumentation im Rahmen der Vorsorge und Begutachtung von Pneumokoniosen.

Ein „Röntgenbild“ entsteht in drei getrennten Teilschritten sowohl bei der Verwendung eines analogen Films als auch bei der digitalen Aufnahmetechnik: nämlich „Belichtung“/Bilderzeugung, -verarbeitung, und -darstellung ( $\bullet$ Tab. 1).

Die Patientenpositionierung und Einblendung des Strahlenganges unterscheidet sich nicht vom Vorgehen bei der analogen Aufnahmetechnik. Die Strahlung wird wie bisher mit Röntgenröhren, die den Qualitätssicherheits-Richtlinien entsprechen, erzeugt.

Nach Auftreffen der Röntgenstrahlen bzw. der Röntgenquanten auf einem „Detektor“ - z.B. Film-Folien-System, Speicherfolie oder digitaler Flachdetektor - wird dort abhängig von der Struktur und Form des durchstrahlten Körpers eine Reaktion ausgelöst, die in Licht (indirekte Konver-

\section{Abstract \\ $\nabla$}

The classification of pneumoconiosis according to ILO standard - comparing a X-ray of the lung with ILO radiographs - is well established in Germany. The extension of digital imaging is a challenging task in occupational medicine as well as in pneumology. Technical requirements are not known sufficiently and the necessary equipment is not well distributed. This paper describes the current position on recording, assessment and documentation of digital imaging of the lung and pleura.

sion) oder elektrische Ladung (direkte Konversion) umgewandelt werden kann. In der digitalen Radiografie werden diese Messdaten (digitale Rohdaten) registriert und daraus das „Röntgenbild“, das sog. Basisbild, das die Grundlage der Bildbefundung ist, errechnet und gespeichert [1-4].

\section{Archivierung und Bildverwaltung}

$\nabla$

In der analogen Bildgebung ist der Film sowohl Informationsträger als auch Speichermedium zur Langzeitarchivierung. Nach der gültigen Röntgenverordnung (RöV) [5] besteht eine Aufbewahrungspflicht für Röntgenbilder von 10 Jahren. Bis 2005 haben die Unfallversicherungsträger (UVT) im Rahmen der sog. Ermächtigung für Vorsorgeuntersuchungen nach Unfallverhütungsvorschrift den Ärzten eine 30-jährige Aufbewahrungspflicht auferlegt. Diese wurde in der Regel auch von den staatlichen Stellen bei Ermächtigung nach Gefahrstoffverordnung (GefStoffV) gefordert. Mit der Novelle der GefStofV vom 23.12.2004 sowie 
Tab. 1 Prinzip der Bildgebung - analog und digital (Ablauf schrittweise von oben nach unten in der Tabelle).

\begin{tabular}{|c|c|c|c|c|c|}
\hline Prinzip & FFS & CR & $\begin{array}{l}\text { DR } \\
\text { direkt }\end{array}$ & $\begin{array}{l}\text { DR } \\
\text { indirekt }\end{array}$ & $\begin{array}{l}\text { CCD } \\
\text { indirekt }\end{array}$ \\
\hline Röntgenstrahl & Röntgenstrahl & Röntgenstrahl & Röntgenstrahl & Röntgenstrahl & Röntgenstrahl \\
\hline Absorptions-Medium & $\begin{array}{l}\text { Verstärker-Folie } \\
\text { (Seltene Erden) }\end{array}$ & $\begin{array}{l}\text { Speicherfolie } \\
\text { Speicher-Phosphor } \\
\text { BaFBr, BaFJ: Eu }\end{array}$ & a-Selen & $\begin{array}{l}\text { Szintillator [s]; } \\
\mathrm{Gd}_{2} \mathrm{O}_{2} \mathrm{~S}\end{array}$ & $\begin{array}{l}\text { Szintillator } \mathrm{Cs} \text {; } \\
\mathrm{Gd}_{2} \mathrm{O}_{2} \mathrm{~S}\end{array}$ \\
\hline Interaktion & Licht & $\begin{array}{l}\text { Elektrische Ladungs- } \\
\text { zustände } \\
\text { (Laser-Readout) }\end{array}$ & $\begin{array}{l}\text { Elektrische Ladung } \\
\left(\mathrm{e}^{-}\right)\end{array}$ & Licht & Licht \\
\hline Messmedium & Film & $\begin{array}{l}\text { Photostimulation } \\
\text { Photodiode und } \\
\text { Photomultiplier }\end{array}$ & Kondensatortechnik & $\begin{array}{l}\text { Photodioden } \\
\text { Matrix (a-Si) }\end{array}$ & $\begin{array}{l}\text { CCD-Chip } \\
\text { Umwandlung Licht in } \\
\text { elektr. Ladung und } \\
\text { Auslesung im CHIP } \\
\text { integriert }\end{array}$ \\
\hline $\begin{array}{l}\text { Verarbeitung, Aus- } \\
\text { lesen der Information }\end{array}$ & $\begin{array}{l}\text { Filmentwick- } \\
\text { lung }\end{array}$ & $A D C$ & $\mathrm{ADC}$ & $\begin{array}{l}\text { TFT-Array } \\
\text { ADC }\end{array}$ & $\begin{array}{l}\text { s. o. } \\
\text { integriert }\end{array}$ \\
\hline Bildwiedergabe & Schaukasten & \#Hardcopy/Monitor* & \#/Monitor* & \#/Monitor* & \#/Monitor* \\
\hline $\begin{array}{l}\text { Archivierung } \\
\text { Dokumentation }\end{array}$ & Filmarchiv & $\begin{array}{l}\text { Filmarchiv } \\
\text { Digitale Medien/PACS }\end{array}$ & Digitale Medien/PACS & Digitale Medien/PACS & Digitale Medien/PACS \\
\hline \multicolumn{3}{|c|}{$\begin{array}{l}\text { ADC - Analog-Digital-Converter } \\
\text { CCD - Charge Coupled Devices } \\
\text { CR - Computerradiography (Speicherfolien-Technik) } \\
\text { DICOM - Digital Imaging and Communications in Medicine } \\
\text { DICOM } 3.0 \text { - aktuelle Version } \\
\text { DR - Direktradiografie } \\
\text { FFS - Film-Folien-System } \\
\text { PACS - Picture Archiving and Communication System } \\
\text { TFT - Thin-Film-Transistor für großflächige elektronische Schaltungen } \\
\text { TFT-Array - flächige Anordnung aus Kondensatoren und Transistoren }\end{array}$} & \multicolumn{3}{|c|}{$\begin{array}{l}\text { amorph - nicht-kristalliner Zustand einer ansonsten kristallinen Substanz } \\
\text { a-Selen - amorphes Selen } \\
\text { a-Si - amorphes Silizium } \\
\text { Array - Anordnung gleicher Elemente in festgelegter Art und Weise } \\
\text { BaFBr/BaFJ: Eu - Bariumfluorbromid- oder Bariumfluorjodidplatte, Europium } \\
\text { dotiert zur photostimulierten Lumineszenz } \\
\mathrm{Cs} \text { - Cäsiumjodid-Kristalle } \\
\mathrm{Gd}_{2} \mathrm{O}_{2} \mathrm{~S} \text { - Gadolinium-Oxysulfit } \\
\text { \#Hardcopy - falls Filmkopie genutzt, ist Filmarchiv erforderlich } \\
\text { Monitor* s. Tabelle }\end{array}$} \\
\hline
\end{tabular}

der Arbeitsmedizinvorsorgeverordnung (ArbMedVV) vom 24.12.2008 wird eine spezielle Aufbewahrungszeit nicht mehr genannt, sodass die nach RöV und Arztrecht übliche 10-Jahre-Regel gilt. Die in der europäischen Richtlinie zur Gesundheitsüberwachung der Arbeitnehmer geforderte Aufbewahrung von Unterlagen zur arbeitsmedizinischen Vorsorge über 40 Jahre muss in Deutschland noch umgesetzt werden.

Mit Einführung der digitalen Bildgebung ergab sich begleitend die Notwendigkeit der digitalen Speicherung und Datenverwaltung. Diese Systeme - PACS ${ }^{1}$ - können jedoch nur ihre volle Effizienz entfalten, wenn alle Aufnahmen (nicht nur die SchnittbildVerfahren) digital angefertigt werden.

Sinnvollerweise muss dann auch die Bildbefundung sowie die Verteilung von Bildern und Befunden elektronisch erfolgen. Innerhalb eines Krankenhauses bietet sich eine WEB-basierte Verteilung an, im Außenverhältnis können elektronische Datenträger, z. B. sog. DICOM-CDs ${ }^{2}$, mit einem standardisierten Datenformat eingesetzt werden. Damit können die vollständigen Bilddaten kostengünstig und verlustfrei digital in Befundqualität zur Verfügung gestellt werden. Außerdem besteht die Möglichkeit, das Bildmaterial in praxiseigene digitale Befundungs- und Archivierungssysteme einzubinden.

Für die digitale Archivierung ist zu unterscheiden zwischen den elektronischen Rohdaten, d.h. Computerdaten ohne Bild, und dem sog. Basisbild, das durch digitale Nachbearbeitung zu einem gewohnten Bildeindruck führt (mit Parametern wie Kontrast, Schärfe und Helligkeit).

Das Basisbild ist vom Arzt, der die Untersuchung durchführt und auch für den Originalbefund zuständig ist, zu archivieren. Nachbearbeitungen seinerseits, die die Diagnose beeinflussen, müs-

\footnotetext{
1 Picture Archiving and Communication System

2 Digital Imaging and Communications in Medicine
}

sen ebenfalls archiviert werden. Alle sonstigen individuellen Veränderungen des Bildcharakters durch einen späteren Betrachter anhand einer CD-ROM oder in einem Intranet entsprechen lediglich seiner persönlichen Interpretation und ändern nicht den primären Befund.

Bislang ist es nicht gelungen, eine langfristige Sicherung der Bilddaten für mehr als 5 Jahre auf einem digitalen Speichermedium zu garantieren. Es ist daher sicherzustellen, dass nicht nur die bislang nach der RöV geforderten 10 Jahre, sondern auch längere Archivierungszeiten im Hinblick auf Latenzzeitschäden realisiert werden müssen $(\bullet$ Tab. 2).

Aufgrund der großen Zahl von Herstellern, unterschiedlichen digitalen Systemen, verschiedensten Computergenerationen und medizinischen Nutzern gab und gibt es wechselnde Schwierigkeiten beim Auslesen und Darstellen der vorgelegten CD-Inhalte. Aus diesem Grunde sah sich die Deutsche Röntgengesellschaft (DRG) im Jahre 2005 nach Klagen der Ärztlichen Stellen, der Bundesärztekammer, verschiedener Berufsverbände und wissenschaftlicher Fachgesellschaften veranlasst, durch die Arbeitsgemeinschaft Informationstechnologie (@GIT) die Initiative „DICOM-CD“ zu starten mit drei wesentlich Zielen:

- Entwicklung einer Spezifikation für Software, mit der DICOM-CDs erstellt werden,

- Entwicklung einer Anleitung für den Umgang mit DICOM-CDs - Entwicklung eines Testverfahrens für DICOM-CDs

Für Radiologen, die mit PACS ausgestattet sind, steht die Lesbarkeit und die Einbindung in die vorhandene Infrastruktur im Vordergrund.

Für die Ärzte, die das Röntgenbild vor allem als Ergänzung und Erweiterung des Befundes insbesondere bei arbeitsmedizinischen Vorsorgeuntersuchungen oder in der Begutachtung von Berufskrankheiten (z. B. zur ILO-Klassifikation) benötigen, ist der Aufwand im Vergleich zum Umgang mit einem herkömmlichen 


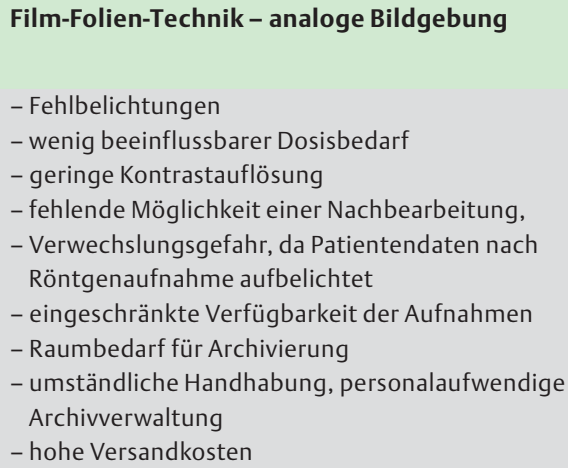

Speicherfolie/Flachdetektoren - digitale
Bildgebung
- direkte Einbindung von Patientendaten und der
Arbeitsliste
- Voreinstellung der Organprogramme
- automatische Aufzeichnung der Expositionsparameter
(CR nicht obligat)
- keine separate Kassettenidentifikation oder
Kassettentransport (außer CR)
- kein Warten zur Kontrolle richtiger Belichtung und
Einstellung
- elektronische Bildverteilung
- vereinfachte interdisziplinäre Visite
- Voraufnahmen rasch verfügbar
- Archiv- und Versandkosten minimiert

Tab. 2 Gegenüberstellung der analogen versus digitalen Bildgebung.
Röntgenbild oder mit einer Hardcopy ${ }^{3}$ nach wie vor $\mathrm{zu}$ hoch. Häufig gelingt es nicht, die mitgebrachte CD so zu öffnen, dass die Daten technisch einwandfrei dargestellt werden.

Von der @GIT werden an Patientendatenträger derzeit folgende Anforderungen gestellt [7-9]:

- Radiologische Bilddaten im aktuellen DICOM-3.0-Format sind Pflicht, weitere Inhalte (z. B. Befunde, Labordaten o. Ä.) sowie ein DICOM-Viewer sind optional erlaubt;

- die CD muss den Anforderungen des DICOM-3.0-Standards entsprechen - insbesondere muss im Stammverzeichnis der CD die Datei „DICOMDIR“ zu finden sein;

- die CD darf keine bösartige Software (Viren etc.) enthalten;

- die CD muss von außen deutlich lesbar beschriftet sein;

- wenn die CD einen Viewer enthält, muss dieser ohne Softwareinstallation und ohne Administratorrechte direkt von der CD laufen können und alle enthaltenen DICOM-Bilder der CD korrekt darstellen können.

Im Rahmen der Befundung berufs- und umweltbedingter Lungen- und Pleuraerkrankungen ist ein sich selbst öffnender DICOM-Viewer auf der CD obligatorisch.

\section{Filmbetrachtungsgeräte|Bildwiedergabe \\ $\nabla$}

Für die analoge Bildbeurteilung ist der Film der Informationsträger. Weitgehend unbekannt unter nicht-radiologisch Tätigen ist die Vorschrift, dass für die Befundung ein Schaukasten (Filmbetrachtungsgerät) nach DIN-Norm 6856-1 (Stand: 10/2007) vorgehalten werden muss, der nach RöV $\S 16$ und der Qualitätssicherungsrichtlinie der Abnahme- und der Konstanzprüfung unterliegt.

Die Digitalisierung in der Radiologie führt aber sowohl aus Qualitäts- als auch aus Kostengründen zur filmlosen Bilderfassung, sodass im Austausch mit den behandelnden Ärzten zunehmend eine $C D$ weitergegeben wird.

In $\S 28$ Abs. 6 der RöV wird gefordert, dass auf Verlangen des weiterbehandelnden Arztes Röntgenbilder inklusive Befund in diagnostischer Qualität weitergegeben werden müssen.

Ärzte, die nicht die Möglichkeiten haben, die Daten einer CD zu lesen, können daher einen Filmausdruck in diagnostischer Qualität (Hardcopy) anfordern.
Die Anforderungen an Bildwiedergabegeräte ${ }^{4}$ in der Heilkunde sind in den Qualitätssicherungsrichtlinien und den Leitlinien der Bundesärztekammer festgelegt [10,6]. Gefordert wird eine objektgerechte Abbildung und zuverlässige Beurteilbarkeit regelhafter und krankhaft veränderter Strukturen, d.h.:

- Die radiologisch gewonnenen Aufnahmen und digital gespeicherten Daten müssen ohne diagnostisch relevanten Informationsverlust wiedergegeben werden.

- Die aufnahmetechnisch eingesetzte Matrix muss in vollem Umfang darstellbar sein; falls dies als Gesamtbild nicht möglich ist, ist durch Zoom-Funktion eine vergrößerte Wiedergabe von Teilbereichen sicherzustellen.

- Werden diagnostische Bilder mit Auflösungsverlust verkleinert (z. B. Speicherung, Kompression, Darstellung mehrerer Bilder auf einem Bildschirm), müssen sie so wiederhergestellt werden können, dass ein Bildpunkt (Pixel) bei der Erstellung identisch dem Bildpunkt bei der Wiedergabe garantiert wird. Die Beurteilung einer Thoraxaufnahme von einer CD-ROM erfordert einen Monitor (Bildwiedergabegerät $=B W G$ ), der die Voraussetzungen zur „Befundung“ erfüllt (DIN V 6868-57). Die Norm unterscheidet zwischen Monitoren der Kategorie A und der Kategorie B ( Tab. 3).

Die technischen Anforderungen für die Befundung von Thoraxaufnahmen (Kategorie A) verlangen eine maximale Leuchtdichte $\left(\mathrm{cd} / \mathrm{m}^{2}\right)>200$, Maximalkontrast $>100$, Matrix des Bildschirmes $\geq 2000 \times \geq 2000$ ggf. $\geq 1000 x \geq 1000$, wenn die Funktion Zoom $1: 1$ (pixeltreu) genutzt wird, und eine Diagonale des BWG $\geq 21$ Zoll für Bildschirme und $\geq 19$ Zoll für Flachbildschirme.

Der Richtwert $\geq 1000 \times \geq 1000$ wird in der Regel durch eine Matrix $1024 \times 1280$ oder $1280 \times 1600$ erfüllt, der Richtwert $\geq 2000 \times$ $\geq 2000$ durch eine Matrix von $2048 \times 2560$. Heute werden üblicherweise Monitore für die Thoraxbefundung mit 3 Mio. Pixeln (entsprechend einer Matrix von $1536 \times 2048$ ) eingeplant. Die weiteren Anforderungen, insbesondere auch Abnahme- und Konstanzprüfungen, Raumbedingungen etc., müssen nach den Leitlinien der Bundesärztekammer und den gesetzlichen Verordnungen $[10,5]$ eingehalten werden.

\footnotetext{
${ }^{3}$ Hardcopy $=$ Digitale Aufnahme auf transparentem Film ausgedruckt, die der Originalqualität entsprechen soll.
}

${ }^{4}$ Monitordarstellung = Bildwiedergabegerät - BWG) 
Tab. 3 Mindestanforderungen an Monitore (Bildwiedergabegeräte - BWG) der Kategorie A und B, modifiziert nach QS-RL.

\begin{tabular}{|c|c|c|c|c|c|c|c|}
\hline \multirow[t]{4}{*}{$\begin{array}{l}\text { Körperregion/ } \\
\text { Methoden }\end{array}$} & \multirow[t]{4}{*}{$\begin{array}{l}\text { Anwendungs- } \\
\text { kategorie }\end{array}$} & \multirow{4}{*}{$\begin{array}{l}\text { Maxim. } \\
\text { Leuchtdichte } \\
\left(\mathrm{cd} / \mathrm{m}^{2}\right)^{2}\end{array}$} & \multirow[t]{4}{*}{$\begin{array}{l}\text { Maximal- } \\
\text { kontrast }\end{array}$} & \multirow{4}{*}{$\begin{array}{l}\text { Matrix des } \\
\text { Bildschirmes } \\
\text { (Richtwerte) }^{1}\end{array}$} & \multirow{4}{*}{$\begin{array}{l}\text { Diagonale des } \\
\text { sichtbaren } \\
\text { Bereiches des } \\
\text { BWG }(\mathrm{cm})\end{array}$} & \multicolumn{2}{|c|}{$\begin{array}{l}\text { Diagonale des BWG nach } \\
\text { Herstellerangabe (Zoll) }\end{array}$} \\
\hline & & & & & & & \\
\hline & & & & & & & \\
\hline & & & & & & CRT & LCD \\
\hline Thorax & A & $>200$ & $>100$ & $\begin{array}{l}\geq 2000 \times \\
\geq 2000\end{array}$ & $\geq 52$ & $\geq 21$ & $\geq 19$ \\
\hline Alle anderen & $\begin{array}{l}\text { B (auch CT } \\
\text { und Durch- } \\
\text { leuchtung) }\end{array}$ & $>120$ & $>40$ & $\begin{array}{l}\geq 1000 \times \\
\geq 1000\end{array}$ & $\geq 34$ & $\geq 17$ & $\geq 15$ \\
\hline $\begin{array}{l}{ }^{1} \text { Für die Befundu } \\
\text { genutzt wird. } \\
2 \text { Der tatsächliche } \\
\text { garantieren. } \\
\text { Weitere Hinweise: }\end{array}$ & es Thorax kann au & n BWG mit einer $\mathrm{k}$ & $\begin{array}{l}\text { eren Matrix ( } \mathrm{z} \\
\text { üblichen im R }\end{array}$ & $\begin{array}{l}\times \geq 1000 \text { ) eingese } \\
\text { andenen Umgebun }\end{array}$ & $\begin{array}{l}\text { verden, wenn die F } \\
\text { eleuchtungsstärke }\end{array}$ & $\begin{array}{l}\text { tion Zo } \\
\text { maxin }\end{array}$ & $\begin{array}{l}\text { ixeltreu) } \\
\text { kontrast }\end{array}$ \\
\hline $\begin{array}{l}\text { Monitore zur Befi } \\
\text { Monitore zur Betı }\end{array}$ & $\begin{array}{l}\text { Ing unterliegen de } \\
\text { tung unterliegen } \mathrm{k}\end{array}$ & $\begin{array}{l}\text { lahme- und Konst } \\
\text { Prüfung; }\end{array}$ & rüfung entsp & DIN 6868-195, Rör & enverordnung und & litätssi & Richtlinie; \\
\hline
\end{tabular}

Tab. 4 Monitorklassen nach Herstellerangaben (M. Wucherer, Nürnberg, pers. Mitteilung 05/2007).

\begin{tabular}{lllll}
$\begin{array}{l}\text { Monitor-Klasse } \\
\text { Mio-Pixel (MP) }\end{array}$ & Matrix & $\begin{array}{l}\text { Auflösung } \\
\text { LP/mm }\end{array}$ & Kontrast & $\begin{array}{l}\text { LD } \\
\mathbf{C d} / \mathbf{c m}^{2}\end{array}$ \\
$1,3 \mathrm{MP}$ & $1024 \times 1280$ & 1,8 & $600-700$ & 700 \\
\hline MP & $1200 \times 1600$ & 2,0 & $700-1000$ & $700-900$ \\
$3 \mathrm{MP}$ & $1536 \times 2048$ & 2,4 & $600-900$ & $600-750$ \\
$5 \mathrm{MP}$ & $2048 \times 2560$ & 3,0 & $600-700$ & $700-800$ \\
$1,3-3 \mathrm{MP}$ & Farbmonitor & & $300-700$ & $180-450$
\end{tabular}

\section{Einfluss auf die ILO-Kodierung}

Derzeit stehen seitens der ILO Standardfilme [11,12] zur Klassifikation der Pneumokoniosen als CD-ROM noch nicht zur Verfügung. In Kürze soll jedoch ein Addendum zur derzeit gültigen Standardversion veröffentlicht werden, das als ILO-2000-D die Standardfilme als CD-Rom verfügbar macht.

Das ILO-Panel hat in seiner Sitzung im März 2008 nachfolgende Vorschläge zur Auswertung und Befundung angenommen:

Die im ILO-2000-System [12] festgelegten Prinzipien zur Beurteilung und Klassifikation analoger Filme oder „Hardcopies“ digitaler Filme gelten unverändert. Hardcopies digitaler Filme sollten nicht kleiner als zwei Drittel der Originalgröße abgebildet werden. Wenn eine Monitor-Befundung (Soft Copy) ${ }^{5}$ und -klassifizierung erfolgt, sollten ILO-2000-D Standardfilme eingesetzt werden.

Sowohl der Patientenfilm als auch der Standardfilm sollten jeweils auf einem Flachbildschirm dargestellt werden, der die Vorgaben der Leitlinien der Bundesärztekammer zur Qualitätssicherung (2007) zur Befundung (Kategorie A) erfüllt. Es handelt es sich um 19" LCD-Monitore. Von der ILO werden derzeit 21" (54 cm diagonal)LCD-Monitore mit $3 \mathrm{MP}$ (Millionen Pixel) ${ }^{6}$, einer maximalen Leuchtdichte von wenigstens 200 Candela $/ \mathrm{m}^{2}$, einer Pixelgröße $\leq 210 \mu \mathrm{m}$ und einer Auflösung von wenigstens 2,5 Linienpaaren/ mm empfohlen. Patientenfilm und Standardfilm sollen simultan in identischer Größe und Leuchtdichte nebeneinander dargestellt werden. Alternative Vorgehensweisen zur Betrachtung, Klassifika-

\footnotetext{
${ }^{5}$ Soft Copy $=$ Digitales Bild, das auf einem Monitor dargestellt ist. Für Befundungszwecke muss ein Monitor mit diagnostischer Qualität der Kategorie A nach Röntgenverordnung verwandt werden.

${ }^{6}$ s. anliegende Tabellen
}

tion und Befundung sind nach dem Beschluss des ILO-Panels auszuschließen.

Beispiele, die nicht empfohlen werden:

- Klassifikation und Befundung der Bilder am PC-Monitor anstelle eines Befundungsmonitors

- Vergleich von Patientenfilm am Monitor mit Standardfilm am Schaukasten

- Klassifikation des Patientenfilms und des ILO-2000-D-Filmes $<2 / 3$ der Originalgröße

- Auswertung mit Papierausdrucken

\section{Die Situation in Deutschland}

Die Gruppe der ZweitbeurteilerInnen der Deutschen Gesetzlichen Unfallversicherung (DGUV) hat sich eindeutig dazu geäußert, dass die Kodierung der Thoraxaufnahme nach der ILO-Klassifikation einen Monitor der Kategorie A erfordert, der die Vorschriften der RöV zur Abnahme- und Konstanzprüfung erfüllt.

Die ILO-Kodierung darf nicht anhand eines Befundberichtes erfolgen. Der für die Klassifikation der Thoraxaufnahme Verantwortliche muss einen Monitor nutzen, der die Kriterien der Befundungsqualität erfüllt. Bei der Monitorbefundung müssen zukünftig die digitalisierten Standardfilme der ILO auf elektronischem Speichermedium (ILO-2000-D) zum Vergleich genutzt und in gleicher Qualität parallel abgebildet werden. Dies erfordert einen zweiten qualitativ gleichwertigen Monitor. Die verwendete Grafikkarte ist den Anforderungen anzupassen. Außerdem muss die Möglichkeit bestehen, dass das genutzte System die parallele Darstellung der ILO-Filme erlaubt. 
An dieser Stelle muss darauf hingewiesen werden, dass derzeit noch einige PACS-Stationen so ausgelegt sind, dass zwar die Voraufnahmen desselben Patienten nebeneinander dargestellt werden können, ein anderer Patient aber nicht dazu eingespielt werden kann, u. a. auch, um Verwechslungen zu vermeiden. Die ILOFilme werden vom System aber als „neuer“ oder „anderer Patient" identifiziert, die parallele Darstellung wird dann verweigert, sodass evtl. diese Einstellung nachgerüstet werden muss.

Die apparativen Voraussetzungen für die entsprechenden Kategorien sind in Tab. 4 zusammengestellt.

Für die Zukunft, d. h. nach dem Vorliegen der digitalisierten Standardbilder der ILO werden mehrere Verfahrenswege als gleichwertig in Betracht kommen:

- Vergleich analoger Thoraxfilm oder Hardcopy mit Standardfilm am Schaukasten;

- Vergleich digitale Thoraxaufnahme mit digitalisiertem ILOStandard an Befundungsmonitoren beim beauftragten Arzt mit der entsprechenden technischen Ausstattung, durch den entsprechend ausgestatteten und fortgebildeten Radiologen, durch spezielle (Befundungs-)Zentren (s.u.).

\section{Praktisches Vorgehen in der derzeit noch bestehenden Situation \\ $\nabla$}

Das Fehlen der digitalisierten Standardfilme ILO-2000-D auf elektronischem Speichermedium wie auch der geforderten technischen Ausstattung führt zunehmend zu Problemen, da die beauftragten Ärzte immer häufiger nur noch CD-ROM und keine Filme mehr erhalten.

Vorübergehend müssen daher die klassische Verfahrensweise des Vergleichs von analogem Bild oder Hardcopy und Standardfilm am Schaukasten und der Vergleich von digitaler Thoraxaufnahme auf einem Befundungsmonitor mit Standardfilm am Schaukasten nebeneinander akzeptiert werden. Letzteres ist auf dem Befundungsbogen zu vermerken ( $\mathrm{T}=$ Monitorbefundung/ Schaukasten).

Der beauftragte Arzt hat die Möglichkeit, technisch entsprechend ausgestattete Zentren (Liste bei GVS bzw. auf der Homepage der GVS) in Anspruch zu nehmen.

Für Fragen zu der Gesamtthematik stehen die Arbeitsgemeinschaft „Diagnostische Radiologie bei arbeits- und umweltbedingten Erkrankungen“ (Vorsitz: Frau PD Dr. K. Hofmann-Preiß, Erlangen) der DRG sowie die Autoren zur Verfügung.

\section{Interessenkonflikte}

$\nabla$

Bei den Autoren besteht kein Interessenkonflikt im Sinne der Rahmenrichtlinie des International Committee of Medical Journal Editors.

\section{Institute}

1 Knappschaftskrankenhaus, Dortmund

2 Institut für Arbeitsmedizin der Heinrich-Heine-Universität Düsseldorf

3 AMD der BG BAU Hannover

4 BG BAU Karlsruhe

Evangelisches Lungenkrankenhaus Berlin-Buch

6 BDT Institut für bildgebende Diagnostik \& Therapie Erlangen

Neuruppin

8 Hermannsburg

9 BG-Klinik für Berufskrankheiten Falkenstein

10 Institut für Arbeitsmedizin und Sozialmedizin, Universitätsklinikum RWTH Aachen University

11 GVS - Gesundheitsvorsorge (vormals: ZAs),Berufsgenossenschaft Energie Textil Elektro, Augsburg

12 DGUV, Sankt Augustin

13 Klinik für Berufskrankheiten Bad Reichenhal

14 Institut für Arbeits- Sozial- und Umweltmedizin der Universität ErlangenNürnberg

15 Berlin

16 Walldorf

\section{Literatur}

1 Ganten M, Radeleff B, Kampschulte A et al. Comparing Image Quality of Flat-Panel Chest Radiography with Storage Phosphor Radiography and Film-Screen Radiography. American Journal of Radiology 2003; 181: $171-176$

2 Garmer M, Hennings SP, Jäger HJ et al. Digital radiography versus conventional radiography in chest imaging: diagnostic performance of a large-area silicon flat-panel detector in a clinical CT-controlled study. Amercan Journal of Radiology 1999; 174: $75-80$

3 Schäfer-Prokop C, Uffmann M, Sailer J et al. Digitale Thoraxradiographie: Flat-Panel-Detektoren oder Speicherfolie? Der Radiologe 2003; 43: $351-361$

4 Zähringer M, Piekarski C, Saupe $M$ et al. Vergleich der digitalen Selenradiographie mit einem analogen Film-Folien-System in der Diagnostik von Staublungenerkrankungen anhand der ILO Klassifikation. RöFo 2001; 173 : $942-948$

5 Richtlinie zur Durchführung der Qualitätssicherung bei Röntgeneinrichtungen zur Untersuchung und Behandlung von Menschen nach $\S \S 16$ und 17 der Röntgenverordnung - Qualitätssicherungsrichtlinie (QS-RL) - vom 20. Nov. 2003 GMBl 2004: 731, geändert durch Rundschreiben vom 28.04.2004, 09.11.2005, 11.01.2006. In: Fiebich M, Westermann K, Zink K RöV \& Co. - Medizinischer Strahlenschutz Vorschriften, Formeln, Glossar. Köln: TÜV Media GmbH, 2007

6 Verordnung über den Schutz vor Schäden durch Röntgenstrahlen. RöV - Röntgenverordnung. Neufassung vom 30.04.2003. In: Fiebich M, Westermann K, Zink K. RöV \& Co. - Medizinischer Strahlenschutz Vorschriften, Formeln, Glossar. Köln: TÜV Media GmbH, 2007

7 Mildenberger P, Kotter E, Riesmeier J et al. Das DICOM-CD-Projekt der Deutschen Röntgengesellschaft - eine Übersicht über die Inhalte und Ergebnisse des Pilottestes 2006. RöFo 2007; 179: 676-682

8 Onken M, Eichelberg M, Riesmeier J et al. Testatprojekt der Deutschen Röntgengesellschaft: Patienten-CDs einfach austauschen. Deutsches Ärzteblatt 2007; 104: 24-26; Supplement Praxis

9 Walz M, Kotter E, Riesmeier J et al. Standardisierung und Testat von DICOM-CDs. Der Radiologe 2007; 47: M82 - M86

10 Bundesärztekammer. Leitlinie der Bundesärztekammer zur Qualitätssicherung in der Röntgendiagnostik - Qualitätskriterien röntgendiagnostischer Untersuchungen - Anlage 2, DKG-Rundschreiben Nr. 63/ 2008, S. 1-6 vom 18.3.2008 http://www.bundesaerztekammer.de/ downloads/LeitRoentgen2008Korr2.pdf; Stand: 23. November 2007

11 Hering KG, Jacobsen M, Borsch-Galetke E et al. Die Weiterentwicklung der Internationalen Staublungenklassifikation - von der ILO 1980 zur ILO 2000/Version Bundesrepublik Deutschland. Pneumologie 2003; 57: $576-584$

12 ILO. Guidelines for the Use of the ILO International Classification of Radiographs of Pneumoconioses. 2000edition (Occupational Safety and Health Series No. 22 [Rev. 2000]). Geneva: International Labour Office, 2002 